\title{
Philosophiques
}

\section{Une double aperception affective et rationnelle de l'homme symbolisant}

\section{Yvan Morin}

Volume 21, numéro 1, printemps 1994

URI : https://id.erudit.org/iderudit/027251ar

DOI : https://doi.org/10.7202/027251ar

Aller au sommaire du numéro

\section{Éditeur(s)}

Société de philosophie du Québec

\section{ISSN}

0316-2923 (imprimé)

1492-1391 (numérique)

Découvrir la revue

Citer cet article

Morin, Y. (1994). Une double aperception affective et rationnelle de l'homme symbolisant. Philosophiques, 21(1), 77-105. https://doi.org/10.7202/027251ar
Résumé de l'article

Une critique de la définition de l'affectivité chez Michel Henry met en évidence le rapport de réversibilité entre la métaphysique de Henry et celle de la philosophie de l'intentionnalité qu'il récuse en vue d'établir une nouvelle conception de l'esprit. Il est alors possible de reformuler le débat en faisant apparaître l'affectivité inhérente à l'action. Pour cela, il s'agit de faire appel à la liberté kantienne et à la perspective symbolique de Cassirer mais en revisant le statut accordé à la sensibilité. Ainsi, la sensibilité se différencie selon son dynamisme propre, l'affectivité, et selon son rapport à la raison, à travers la perception qui identifie seulement de façon indirecte (contrairement à ce que laisse entendre Henry) la sensibilité aux sens. Il s'agit d'un nouveau rapport entre le sensible et l'intelligible. 


\title{
UNE DOUBLE APERCEPTION AFFECTIVE ET RAIIONNELIE DE L'HOMME SYMBOLISANI
}

\author{
par \\ Yvan Morin
}

\begin{abstract}
RÉSUMÉ : Une critique de la définition de l'affectivité chez Michel Henry met en évidence le rapport de réversibilité entre la métaphysique de Henry et celle de la philosophie de l'intentionnalité qu'il récuse en vue d'établir une nouvelle conception de l'esprit. Il est alors possible de reformuler le débat en faisant apparaître l'affectivité inhérente à l'action. Pour cela, il s'agit de faire appel à la liberté kantienne et à la perspective symbolique de Cassirer mais en revisant le statut accordé à la sensibilité. Ainsi, la sensibilité se différencie selon son dynamisme propre, l'affectivité, et selon son rapport à la raison, à travers la perception qui identifie seulement de façon indirecte (contrairement à ce que laisse entendre Henty) la sensibilité aux sens. Il s'agit d'un nouveau rapport entre le sensible et l'intelligible.
\end{abstract}

ABSTRACT: The critic of Henry's definition of affectivity shows that the metaphysics of Henry is the mirror of the metaphysics carried by the philosophy of intentionality. By challenging the last one, Henry aims to establish a new conception of the spirit. I suggest that there is more to find about affectivity if we look at it in the context of action. The kantian liberty and the symbolic perspective of Cassirer are going in this way if we accept to change the status of the sensibility. In fact, it is possible to differentiate the sensibility : the affectivity enunciates the dynamism of the sensibility and, by his relation to the reason through the perception, shows only indirectly its identification to the sense. Then, we find a new relationship between the sensibility and the intelligibility.

Henry affirme que « ce qui se sent sans que ce soit par l'intermédiaire d'un sens est en son essence affectivité » (L'essence de la manifestation, p 577). Par l'affectivité, il propose une nouvelle 
conception de l'esprit et, par l'exclusion des sens ${ }^{\mathrm{I}}$, il en propose une phénomenologie où la manifestation trouve son essence en cette affectivité qui, à la fois, échappe à la philosophie de l'intentionnalitè et met en question sa conception de l'esprit ${ }^{2}$. Je propose d'apercevoir, dans l'affectivité, le dynamisme spécifique de la sensibilité afin de pouvoir s'interroger aussi sur le rapport de la sensibilité à elle-même selon qu'elle s'exprime affectivement, en préorganisant les sens avant toute exclusion de ceux-ci, ou selon qu'elle s'exprime à travers les sens, en tant qu'ils sont rendus disponibles à la perception et à la représentation rationnelle avant que l'esprit soit modifié de telle sorte qu'il soit rendu lui-même affectif.

Il s'agirait, par l'affectivité, de rétablir le statut de la sensibilitė dans la conception de l'homme. Une double aperception affective et rationnelle de l'homme, pour rendre compte de la spécificité respective du sensible et de l'intelligible, permettrait de s'interroger sur la réduction rationnelle de la sensibilité aux sens, non seulement chez Henry mais aussi chez Kant, où elle s'effectue empiriquement tout en faisant de l'affectivité une pathologie affligeant la raison ${ }^{3}$. De même, l'expression symbolique de l'intelligible, chez Cassirer, parviendrait à proposer une nouvelle conception de l'esprit au niveau même de

I. Cette exclusion des sens et, à travers eux, de la sensibilité elle-même vaut pour tout contenu sensible, au sens que lui donne Henry, " qu'il s'agisse d'un contenu empirique ou d'un contenu pur » (L'essence de la manifestation, Paris. P.U.F., I963. p. 579). En ce sens, l'hétérogènéité ou l'opposition entre l'affectivité et la sensibilité implique aussi la proposition d'un contenu sensible interne en général, puisque « le sentiment n'est jamais et ne peut être senti » (ibid., p. 579). En effet, l'intégration de l'être et du Soi s'effectue par « ce qui se sent soi-même, de telle manière qu'il n'est pas quelque chose qui se sent mais le fait même de se sentir ainsi soi-même » (ibid., 58I). C'est par ce fait que l'affectivité peut s'imposer comme conception de l'esprit, puisque se sentir, cest sentir le Soi.

2. Cet apport philosophique me semble important parce qu'il pourrait permettre de modifier l'aperception que l'homme peut avoir de lui-même en faisant apparaître. à travers l'affectivité, l'altérité essentiellement sensible que la raison rencontre en l'homme. Il ne serait pas question d'un a-rationnel où le mythe s'affirme sans la raison, d'un irrationnel auquel s'oppose la raison, d'une préfiguration de la rationnalité qui se vise elle-même téléologiquement ou d'un préréfléchi repris réflexivement, mais bien d'affectivité. Cependant. Henry n'aperçoit pas, dans l'affectivité, l'altérité fondamentalement sensible que la raison rencontre en l'homme mais une nouvelle conception de l'esprit qui nous renferme dans cette raison.

3. La raison kantienne fait place à la sensibilité mais ne la formule que selon cette raison. D'où le privilège accordé à la formulation empirique de la sensibilité par la Critique de la raison pure, pour rendre compte des formes a priori de la sensibilité. et le caractère pathologique de l'affectivité selon la Critique de la raison pratique. Le 
l'esprit, dans la mesure où cet esprit s'enracine dans l'affectivité, au sens sensible $^{4}$ et non pas spirituel que lui donne Henry. En effet, la symbolisation peut apparaître et se définir comme l'ouverture a priori de la raison à l'affectivité, au sein de l'aperception, de telle sorte que la raison n'apparaît, depuis cette affectivité, que selon un décentrement a priori. Enfin, dans la mesure où l'action s'éprouve elle-même, comme une réciprocité sensible finie articulant l'altérité inhérente à tout ce qu'elle implique concrètement, et s'éprouve jusque dans la totalité symbolique de ce qu'elle met ainsi en tension, l'affectivité apparaît comme une passivité inhérente à l'action et non comme une passivité séparée d'une activité et d'une totalité proprement rationnelles. Ainsi, l'action ${ }^{5}$ per- $^{-}$ met de reprendre le questionnement de Kant et de Cassirer sur le rapport entre le sensible et l'intelligible mais en préservant le dynamisme

sentiment est « pathologique » (Kant, Critique de la raison pratique (1788), trad, Luc Ferry et Heinz Wismann, F. Alquié (dir.), Paris, Gallimard, p. Iog), sauf dans le cas unique où il n'a pas d'existence propre mais seulemement comme « effet de la conscience de la loi morale » (ibid., p. Iog). Le sentiment moral est un sentiment produit intellectuellement et c'est en tant que tel que le rationalisme kantien en a une connaissance a priori.

Je propose, en concevant l'affectivité comme le dynamisme spécifique de la sensibilité, d'élargir la place accordée par Kant à la sensibilité, en même temps, par le rapport indirect de la raison kantienne à la sensibilité, j'aperçois, au niveau intelligible, l'esquisse du symbole. Les symboles, comme « présentations indirectes » (Kant, Critique de la faculté de juger, I79o, trad. A. J.-L. Delamarre, J.-R. Ladmiral, Marc B. de Launay, J.-M. Vaysse, Luc Ferry et Heinz Wisman, F. Alquié (dir.), Paris, Gallimard, 1985. P. 314) des concepts pensés par la raison, nous renvoient alors à la symbolisation elle-même dont l'expression intelligible, en étant reliée à l'affectivité, se distingue de celle de la raison et de ses opérations conceptuelles qui lient le sentiment à des sources empiriques indépendamment de la préorganisation affective des sens.

4. Cassirer reste cependant prisonnier du privilège que le rationalisme accorde à la formulation empirique de la sensibilité, d'oủ l'exigence d'une trace empirique pour soutenir l'élaboration des formes symboliques. En effet, Cassirer substitue l'« animal symbolicum 》 (Essai sur lhomme, trad. N. Massa, Paris, Minuit, 1975, p. 44) et non une affectivité symbolisante à l'animal raisonnable. Aussi, il ne peut dégager la symbolisation elle-même de la multiplicité formelle, dans La philosophie des formes symboliques, faute d'une aperception affective par laquelle la raison rencontre a priori l'altérité en l'homme et se trouve, par là, décentrée, énonçant ainsi cette symbolisation et l'aperception duale (affective et rationnelle) par laquelle elle s'articule en l'homme.

5. L'action est, en ce sens, distincte de l'effort biranien et de l'opposition de « l'individualité de l'être de l'ego à la conception empirique de l'individuation spatio-temporelle » (Henry, 1987, I42). En effet, l'espace-temps s'exprime, ici, subjectivement, au sens d'une aperception affective, même s'il se prête à une conception 
spécifique de cette sensibilité, l'affectivité, dans l'aperception de l'homme par lui-même.

Je me propose de resituer la définition de l'affectivité que donne Henry dans le contexte de L'essence de la manifestation, où il la formule, afin de pouvoir entreprendre une critique de cette définition, autant en rapport avec la métaphysique de l'intentionalité qu'il combat qu'en rapport avec l'affectivité inhérente à l'action.

\section{La position et le projet de Henry}

Henry s'inspire de la problématique de Maine de Biran mais il apporte à celle-ci une contribution originale. Comme l'écrit Henry, dans Philosophie et phénoménologie du corps (p. $\left.220^{-221}\right)^{6}$, il n'existe pas de théorie de la vie affective, c'est-à-dire de théorie ontologique de la passivité, chez Maine de Biran. En effet, Maine de Biran nous entretient du « sentiment qu'a la force motrice de son existence absolue ${ }^{7}$. Aussi, il associe le sentiment à l'effort musculaire au lieu de les dissocier et d'énoncer le sentiment d'un point de vue strictement affectif, comme le propose Henry. Corrélativement, chez Maine de Biran, le sentiment n'exclut pas la sensation puisque « la sensation est naturellement conjointe avec l'étendue du corps propre, dont le moi se distingue sans pouvoir se séparer » (ibid, p. 212). Cependant, malgré ces différences, Henry et Maine de Biran partagent un présupposé commun par lequel ils s'opposent à Descartes : c'est l'identité du moi au corps et la distinction de cette identité par rapport au corps mais sans que celle-ci puisse s'en séparer. Chez Henry, la transcendance instaurée par cette séparation trouve son fondement dans l'affectivité. Ainsi, Henry écrit que «le corps est cette réalité hétérogène à la pensée pure qui, agissant sur elle, la rend affective » (L'essence, op.cit., p. 642). C'est en ce sens qu'il est question d'une nouvelle conception de l'esprit correspondant à l'affectivité elle-même ${ }^{8}$. De plus, cette affectivité se loge dans le corps puisque c'est

empirique propre à une aperception rationnelle : l'espace, au sens d'une réciprocité sensible finie, s'aperçoit affectivement et se temporalise symboliquement par la totalité mise en jeu dans l'action. Aussi l'affectivité symbolisante est un rapport entre le sensible et l'intelligible qui se distingue de celui qui est instauré par l'animal raisonnable.

6. Philosophie et phénoménologie du corps : essai sur Pontologie biranienne, Paris, P.U.F., $2^{\mathrm{e}}$ éd. Paris, 1987.

7. Maine de Biran (Marie François Pierre Gonthier de Biran), Euures choisies, introd. H. Gouhier, Paris, Aubier-Montaigne, 1942, p. 212.

8. Chez Descartes, le cogito s'identifie à l'entendement et s'affinme comme substance distincte de la substance corporelle. Chez Maine de Biran, il s'identifie à la volonté 
par cette hétérogénéité du corps à la pensée que Maine de Biran propose « un corps qui est subjectif et qui est l'ego lui-même », selon les termes mêmes de Henry ${ }^{9}$. En somme, les deux auteurs insistent sur l'être de l'ego et font de l'aperception immédiate de cet ego le critère de toute vérité métaphysique. Ainsi, Henry écrit : «Laffectivité est révélation de son être et c'est pourquoi elle est l'être » (L'essence, op. cit., p. 668). Cependant, ce choix d'une métaphysique de la singularité plutôt que de l'intentionnalité s'affirme, par rapport à cette dernière, comme une métaphysique anti-métaphysique. La singularité s'identifie, alors, à une individualisation de la pensée par le corps afin de formuler le projet « d'instituer une élucidation systématique de l'essence de l'affectivité » (ibid., p. 84I). Je cerne cette position et ce projet avant de proposer une critique faisant place à une singularité inhérente au dynamisme, non de l'esprit, mais de la sensibilité qu'est l'affectivité et à la symbolisation, comme nouvelle conception de l'esprit, l'accompagnant lors de l'action.

\subsection{La position : une métaphysique de la singularité}

Selon Henry, l'affectivité révèle son être et, pour cette raison, elle est l'être. Le mouvement de l'essence de la manifestation est double : la manifestation consiste dans la réalité de l'acte d'apparaître et non dans l'apparence elle-même. Ceci se justifie dans la mesure où l'essence n'est que là où est la détermination procurée par notre existence, au sens corporel, tout en se distinguant immédiatement de cette détermination par laquelle elle est mais sans pouvoir s'en séparer. En d'autres mots, L'essence de la manifestation s'énonce comme affectivité inhérente à la manifestation. C'est en tant que l'affectivité est l'essence de l'essence, liée à

et s'affirme comme mode de l'auto-révélation de l'ego, qui, à la fois, s'aperçoit dans l'effort et se distingue du mode de révélation qu'implique la résistance du corps dans cet effort. Il s'énonce comme « affectivité de la pensée », chez Henry (Philosophie, op.cit., p. 197), et se trouve lié au corps subjectif dont « activite et passivité sont bien plutôt deux modalités différentes » (ibid., p. 226) de telle sorte que la passivité n'est pas subordonnée à l'activitè. Le rapport de Henry à Maine de Biran réside dans cette reprise du corps subjectif et dans la prise en compte de la passivité afin de rapporter la distinction elle-même entre la passivité et l'activité à son fondement, tel que Henry l'expose par sa théorie ontologique du corps. En somme. la subjectivité va de la pensée cartésienne vers le corps biranien, au sens actif, puis retourne du corps, au sens passif, vers la pensée qu'il contraint, chez Henry. C'est le processus réflexif, inhérent à ce troisième moment, qui permet à Henry de fonmuler l'ipséité du sentiment et de la formuler de telle sorte qu'il s'agisse d'une nouvelle conception de l'esprit.

9. M. Henry, Philosophie et phénoménologie du corps : essai sur l'ontologie biranienne, Paris, P.U.F., $z^{\mathrm{e}}$ éd., Paris, 1987 , p. 15 . 
l'auto-suppression de l'essence pure et abstraite de l'intentionnalité, que la manifestation réside dans l'acte d'apparaître et non dans l'apparence. Ce rapport entre l'affectivité et la manifestation est la façon de Henry d'énoncer la démarche phénoménologique en tant qu'elle porte sur la différence ontologique entre son être (plutôt que l'être) et l'étant (qu'il reçoit). Voyons donc de plus près cette double remise en question de la philosophie de l'intentionnalité ${ }^{\text {To }}$ qui s'effectue à travers l'effectuation affective de l'essence et à travers cette auto-affectation par laquelle s'énonce la manifestation.

L'effectuation affective de l'essence démontre, en se suffisant à elle-même en son être, qu'elle est aussi l'auto-suppression de l'essence pure et abstraite de la philosophie de l'intentionnalité qui prétend énoncer l'être. Le souffrir est le sentiment au fondement du sentiment de soi en tant qu'il indique, non pas le sentiment d'impuissance qui s'énoncerait intentionnellement, mais l'impuissance du sentiment qui est inhérente à la passivité originelle à l'égard de soi où l'être est livré à lui-même. La toute-puissance de cette impuissance du sentiment s'effectue dans la contemporanéité de la joie vers laquelle se dépasse la souffrance : « en tant que celui qui souffre se trouve renvoyé de par sa souffrance même à ce qui en lui supporte cette souffrance, à luimême » (ibid., p. 846). Ainsi, l'affectivité est l'essence originaire de la révélation de l'être où souffrance et joie renvoient au fond de l'unité de l'être avec soi plutôt que de s'opposer : « dans l'existence le contraire ne procède pas de l'opposition mais de l'identité » (ibid., p. 844), c'est-àdire « la souffrance se dépasse vers la joie, en tant qu'elle révele l'absolu » (ibid., p. 847).

De même, la philosophie de l'intentionalité se trouve remise en question au niveau de l'étant du fait que c'est « comme objet que l'étant est reçu » (ibid., p. I56), c'est-à-dire que l'étant se manifeste. Le sens, ce vers quoi se dépasse l'existant, passe par l'objet et celui-ci n'est pas l'étant mais l'étant reçu. Il apparaît que, selon Henry (ibid., p. 741), le sentiment à l'égard de ce qui est reçu est aussi et avant tout une

ı. Henry dialogue principalement avec Heidegger, en particulier à partir de la problématique cartésienne dont Henry reprend le questionnement dès le début de L'essence de la manifestation. Henry s'interroge sur le sens de l'être de l'ego tandis que Heidegger s'interroge sur le sens de l'être du «sum »dans le «cogito sum ». Chez Henry, l'affectivité fonde la compréhension heideggérienne de l'horizon ontologique du monde. Le traitement du « problème de l'"affectivité intentionnelle" » (L'essence, op. cit., p. 598-6og) précise ce rapport. Cependant, il n'est seulement question de Heidegger mais aussi d'un rapport à la philosophie moderne et contemporaine. 
manière de se sentir, un sentiment de soi qui est « un sentiment du Soi » (ibid., p. 58I). C'est en son être même que, pour l'affectivité, il est question de l'être au sein de l'étant. Ainsi, l'affectivité, comme sentiment éprouvé de son être, livre l'être à soi, c'est-à-dire à une absence de dépassement. Cette absence de dépassement ontologique est justement ce qui dépasse ontologiquement le sentiment éprouvé et ce qui l'affecte, comme le fait remarquer Henry (ibid., p. 590, 840). Le dépassement ontologique est, pour l'affectivité, identique au non-dépassement de son être. Toute transcendance se retient en cette immanence qui en est l'essence. Ainsi, le temps est cette auto-affection que détermine l'affectivité puisque c'est à partir de soi, de son propre surgissement, que l'affectivité détermine ce qui l'affecte, c'est-à-dire l'objet en tant qu'il est l'étant reçu et non l'étant. La phénoménalité tient dans ce pouvoir de réception de l'étant par lequel l'être se réalise intérieurement comme cet acte de saisie lui-même qui se révèle. Il ne s'agit donc pas d'un pouvoir d'abstraction de ce qui est saisi dans un horizon de compréhension puisque le sentiment à l'égard de ce qui est reçu ne se constitue qu'en étant aussi et avant tout une manière de se sentir qui se radicalise en sentiment du soi ou, encore, dans le soi propre au sentiment. Cette révélation originaire de l'affectivité à elle-même et cette constitution de la manifestation comme son contenu s'intègrent en un projet, celui de Henry.

\subsection{Le projet d'une problématique unissant l'affectivité et l'affection tout en excluant la saisie intentionnelle qui les sépare}

Comme l'écrit Henry : « Le projet d'instituer une elucidation systématique de l'essence de l'affectivité a trouvé son expression dans la délimitation de deux questions fondamentales visant la nature de la révélation originaire qui s'accomplit en elle et la constitue, visant le comment et le contenu de cette révélation » (ibid., p. 84I).

Le projet de Henry réside dans cette problématique telle qu'elle se met en place depuis sa position métaphysique sur la singularité. Cette problematique inverse la relation habituelle entre l'affectivité et l'affection en l'ancrant à la fois dans le rapport de fondation les unissant et dans l'exclusion de la saisie intentionnelle où ce rapport de fondation se néantise et se donne comme monde. Selon cette problématique, le rapport de fondation réside dans le fait que c'est l'affectivité qui détermine ce qui nous affecte tandis que l'anéantissement, qu'il s'agit d'exclure, réside dans le rapport inverse par lequel l'intentionnalité prétend que c'est l'affection qui détermine l'affectivite ${ }^{\mathrm{II}}$.

II. Cest ici que les présupposés de Henry jouent pleinement et se démarquent de la problématique que je propose. Henry propose l'affectivité comme une nouvelle 
C'est dans cette perspective que, pour Henry, la nouvelle conception de l'esprit qu'est l'affectivité inclut la sensibilité en la réduisant aux sens (tout en excluant ces sens de la définition explicite de l'affectivité) et exclut le rapport inverse d'inclusion que la philosophie de l'intentionnalité tenterait d'établir selon lui. Précisément, l'affectivité, comme nouvelle conception de l'esprit, n'est jamais sensible alors que la sensibilité, en tant qu'elle est identifiée aux sens, est toujours ou « constamment affective » (ibid., p. 6oo). Cette problématique paradoxale évolue de ce «jamais », par lequel s'énonce le projet en tant qu'il réalise sa visée du « comment », à ce « toujours », par lequel ce projet réalise sa visée du « contenu ». En effet, l'auteur affirme que « ce qui se sent sans que ce soit par l'intermédiaire d'un sens est en son essence affectivité » (ibid., p. 577) puis il livre le combat là où « la thèse fondamentale de la problematique selon laquelle un sentiment ne saurait être perçu ni senti est en question » (ibid., p. 783). Selon la problématique de l'auteur, le sentiment est une intuition qu'aucune intuition n'est susceptible de remplir. Perception, reproduction, représentation, etc, du sentiment demeurent des significations idéales, irréelles puisque, à travers ces actes, la conscience n'éprouve jamais réellement le sentiment qu'elle se donne mais au contraire le détruit et s'auto-détruit du point de vue de son effectuation phénoménologique. Ainsi, le sentiment représenté n'est pas réellement un sentiment puisqu'il est renvoyé à ce qui le remplace au lieu d'être donné dans son affectivité, c'està-dire d'être vécu. Ultimement, c'est parce que le sentiment s'éprouve qu'il peut ensuite se proposer en ce milieu transcendant de la représentation, de la perception, du sentir... où il ne peut se proposer du point de vue de son effectuation phénoménologique. En somme, le néant, par lequel le monde et la sensibilité y ayant cours s'effectuent, ne peut s'effectuer, ontologiquement, que par l'affectivité qui lui est antérieure et qui le rend possible.

Dès lors, tout comme l'affectivité s'énonce comme une essence de l'essence lièe à l'auto-suppression d'une essence pure et abstraite, la

conception de l'esprit, au lieu d'y apercevoir un rétablissement de l'intégritè de la sensibilité elle-même. Il privilégie aussi le postulat rationaliste réduisant la sensibilité aux sens, au lieu de soulever le problème de la transformation du statut de la sensibilité. Cette transformation peut s'effectuer à travers la différenciation de la sensibilité qui permet d'établir le rapport entre l'affectivité et les sens et qui s'énonce comme rapport respectif entre cette sensibilité s'exprimant elle-même comme elle-même, par l'affectivité, et cette sensibilité s'exprimant dans un rapport à la raison, à travers la perception qui identifie seulement de façon indirecte la sensibilité aux sens. 
conceptualisation s'énonce de telle sorte que la nouvelle conception de l'esprit apparaisse. Concevoir le sentiment consiste, dans un sens premier, dans la manière de se le donner et de le recevoir. Concevoir, c'est alors désigner, expliciter, montrer; cela n'ajoute rien à l'essence du sentiment. Cette première conception est liée à une seconde qui se supprime puisque concevoir c'est, dans ce cas, dé-réaliser la réalité du sentiment et, en même temps, le réaliser de nouveau à un niveau abstrait comme représentation dans un milieu transcendant. Or le sentiment représenté n'est pas réellement un sentiment puisqu'il est renvoyé à ce qui le remplace au lieu d'être vécu. Aussi, le sentiment représenté renferme en lui-même l'auto-suppression de l'ancienne conception de l'esprit qu'énonce l'intentionnalité et fait place à la nouvelle conception de l'esprit que propose Henry par l'affectivité inhérente au sentiment vécu. Cependant, les deux types de conception de l'affectivité semblent être l'envers exact l'une de l'autre de telle sorte qu'elles s'énoncent depuis un même rapport conceptuel, entre l'unité et l'opposition, pour rendre compte de façon métaphysique du rapport entre le corps et l'âme pensante en l'homme.

\section{Une critique de la définition de Yaffectivité chee Henry}

\subsection{La réversibilité entre la métaphysique de la singularité que propose Henry et la métaphysique de l'intentionnalité à laquelle il s'oppose}

Il semble possible de dire de Henry, ce que Gouhier écrit au sujet de Maine de Biran, en cernant son rapport avec Descartes : « en supprimant le problème de l'union de l'âme avec le corps, Maine de Biran fait rebondir celui de leur séparation » (cEuvres choisies, op. cit., p. 37). De même, la métaphysique de la singularité de Henry se formule comme le problème d'un pouvoir d'union de l'affection à l'affectivité. Cette union disparaît dans le rapport fusionnel à elle-même mais en faisant aussi rebondir le problème de la séparation de l'affection par rapport à l'affectivité que véhicule la métaphysique de l'intentionnalité. De fait, Henry fonde le problème de la séparation dans une union différenciée, au lieu de seulement le faire rebondir ${ }^{12}$. Aussi, selon Henry, « la

12. Par là, il redouble non seulement l'union, pour y inclure la séparation, mais aussi la séparation, en la rendant séparable d'elle-même. Ainsi, Henry intègre réflexivement en un seul, le fait primitif qui risque de se scinder en deux, chez Maine de Biran, du fait qu'il découvre successivement ses deux aspects : « Le premier fait primitif était celui de l'union de l'âme et du corps, le second exprime l'expérience de leur séparation, la liberration de l'âme rendue à la vie absolue de l'esprit » (Philosophie, op. cit., p. 242). Cependant, l'approfondissement de la pensée de Maine de Biran, que Henry recherche, apparaît indissolublement lié à un redoublement dont la réflexivité appartient seulement à Henry. 
possibilité même de l'opposition réside au contraire dans l'unité (concrète) » (L'essence, op., cit., p. 779). Dès lors, la métaphysique de la singularité reste profondément liée à la métaphysique de l'intentionnalité et, du point de vue de cette dernière, la première ne peut qu'exclure à son tour cette métaphysique de l'intentionnalité qui l'a déjà exclue par avance pour s'affirmer elle-même.

En effet, la transcendance mise en jeu par l'intentionnalité, même si elle « ne fonde jamais le caractère souffrant » (Henry, L'essence, op. cit., p. 752) de la séparation dont souffre la nostalgie ${ }^{13}$ en son être-éprouvé, fonde, néanmoins, la séparation dont souffre cette nostalgie, puisque l'intentionnalité s'énonce comme pouvoir de séparation. Pouvoir de séparation dont elle ne peut se séparer sans se séparer d'elle-même, la saisie intentionnelle apparaît pour ce qu'elle est dans ce manque à être, dans cette souffrance alors qu'il lui est porté atteinte en son pouvoir de séparation. Nostalgique d'elle-même, elle cherche son mode d'être spécifique et son expression de la joie et de la souffrance qui sont atteints lorsqu'est atteint l'être par lequel elle est.

Ainsi, de façon intentionnelle, je souffre d'être ici alors que je désire être ailleurs et, en même temps, j'éprouve la joie de me retrouver, de me reconnaître dans l'ailleurs où tout se maintient dans la fête de mes retrouvailles par et dans l'ailleurs d'où s'établit le rapport originaire à moi-même que cet ailleurs dépose en moi alors que je me tends vers lui. Tel est l'élan passionné et passionnel de la saisie intentionnelle. Cela peut, aussi, se faire de façon méthodique et froide, en s'inspirant du rapport cartésien de l'homme avec Dieu, ou encore d'une façon dite éthique par le rapport métaphysique que Lévinas ${ }^{\mathrm{I}}$ établit de l'homme à l' Autre. Que le rapport de l'homme soit à Dieu, à l'Autre, à l'Ailleurs..., dans tous les cas il s'agit de différences de modalités d'une même saisie intentionnelle de ce qui èchappe a priori à cette saisie intentionnelle. Ce

13. Henry maintient, à travers sa critique, que l'être transcendant éveille notre souffrance et il cite Heidegger : « La nostalgie, dit Heidegger, est la douleur que nous cause la proximité du lointain » (ibid., p. 752). Ce qu'apporte Henry, c'est le lien par lequel l'affectivité, comme immanence, fonde la transcendance. Cependant. Henry a déjà transformé la nostalgie de l'être, de telle sorte que l'être visé par la connaissance, comme ce qu'elle ne peut pas être et ce dont elle est séparée, devient l'être qui se sépare de lui-même pour être « sa propre nostalgie » (ibid. p. gol. Or, l'être est sa propre nostalgie parce que, en même temps, en ce qui concerne la connaissance, la saisie intentionnelle est nostalgique d'elle-même en ne parvenant plus à être.

14. Ainsi, le visage est « la manière dont se présente l'Autre, dépassant lidée de l'Autre en moi » (Totalité et infini. Essai sur l'extériorité, La Haye, Martinus Nijhoff. 1971, p. 2I). L'infinition remplace l'infini par lequel l'homme cartésien a l'idée de Dieu en lui. 
rapport intentionnel de la pensée à l'impensable peut se faire aussi selon différentes modalités temporelles. Par exemple, anticiper un évènement qui ne se produit pas peut mener à la dépression de la fête, c'està-dire à la dépression de mes retrouvailles avec moi-même dans l'ailleurs, qui, en n'ayant pas eu lieu, ne laisse qu'un moi livré à luimême et s'identifiant seulement alors au corps de façon stricte. En somme, le message essentiel de la saisie intentionnelle est l'inverse exact de la métaphysique de la singularité que propose Henry. Depuis l'être, le sujet s'adresse à lui-même comme étant en se disant : je ne peux éprouver ton être à ta place mais je peux t'aider à l'éprouver en te procurant l'être par lequel il s'énonce généralement afin de pouvoir aussi s'énoncer singulièrement, voire s'énoncer au sein même de cette singularité. Ainsi, l'horizon ontologique d'un monde ne se déploie que par son néant qui se creuse au sein de l'étant lui-même et non plus « dans l'être de l'existant » (Heidegger, « Qu'est-ce que la métaphysique?», trad. H. Corbin, dans Questions I, Paris, Gallimard, ig68, p. 63). Dès lors, il ne s'agit plus de s'opposer à une transcendance qui nous présente la métaphysique en nous retenant dans le néant par l'angoisse, mais de préserver par l'humour cet apparaître ontologique d'une transcendance s'énonçant par le néant : l'être se donne à lui-même en apparaissant comme être là même oủ il semble ne pas pouvoir être, c'est-à-dire là où se trouve ce que nous appelons le corps, et en étant concerné par cela, c'est-à-dire par l'étant tel qu'il s'aperçoit à travers cet espace. Cette intégration de l'espace et du corps lui-même, s'éprouverait dans l'action, comprise au sens d'un processus d'incarnation.

\subsection{De la critique de l'antinomie, entre ces deux types de métaphysique, à l'affectivité inhérente à l'action}

Je propose de considérer l'affectivité dans le contexte de l'action ${ }^{15}$ telle qu'elle s'éprouve elle-même, comme réciprocité sensible, jusque dans la totalité symbolique qu'elle met ainsi en tension. Par l'affectivité inhérente à l'action, la sensibilité est irréductible non seulement à la formulation rationnelle de celle-ci à travers les sens, lorsqu'ils sont rendus pré-disponibles à la perception, mais aussi à l'hétérogénéité entre l'affectivité et les sens, par laquelle Henry prétend, à la fois, faire de l'affectivité une nouvelle conception de l'esprit et réduire la sensibilité aux seuls sens, tout comme le propose le rationalisme inhérent à la

15. Cette critique vise l'élargissement de la conception de la pratique humaine, à laquelle Kant nous introduit, afin de faire apparaître l'affectivité inhérente à l'action, par les modifications de la problématique kantienne qui ont été annoncées au début du présent texte. Par l'auto-destitution symbolique de la 
philosophie de l'intentionnalité. Une telle conception de l'affectivité implique au moins trois conditions à satisfaire : une conception de l'affectivité comme dynamisme spécifique de la sensibilité, le rétablissement de la sensibilité dans l'aperception, que cela implique a priori, et l'articulation de l'aperception duale (à la fois affective et rationnelle) par l'horizon symbolique de l'action.

Premièrement, l'affectivité comporte un dynamisme sensible qui lui est propre et elle est même le dynamisme spécifique de la sensibilité autant affective que sensorielle, en tant qu'elle préorganise ${ }^{16}$ les sens avant de les libérer, en les rendant disponibles à leur conception empirique par la raison kantienne, et avant de se limiter à ce qui se sent sans que ce soit par l'intermédiaire d'un sens, chez Henry. Ainsi, la préorganisation affective des sens s'exprime par la mobilité de l'horizon symbolique intégrant tout ce que l'action met existentiellement en tension. Par exemple, l'outillage, à travers lequel j'accomplis ma tâche, implique l'outil que j'utilise et lui donne son sens en articulant, au sein de cette totalité que je mets en oeuvre, mon rapport à cet outil, dans un espacetemps donné. Cet espace-temps donné s'énonce empiriquement et, par là, objectivement ${ }^{17}$ en se formant a priori dans l'esprit, au sens d'une aperception rationnelle, parce qu'il donne accès à l'espace-temps de

transcendance qui se trouve dans le transcendantal, il devient possible de faire place à l'immanence qu'il comporte et d'élargir la place faite à cette immanence. La façon d'articuler le rapport entre l'immanence et la transcendance est, donc, inverse de celle de Henry. Cependant, au lieu de chercher à savoir lequel est l'envers de l'autre, j'adopte d'emblée la position d'une réciprocité par laquelle se développe ce rapport inverse. Cette réciprocité est sensible et elle est sensible en incluant dans un rapport symbolique ce qui lui reste étranger afin que la subjectivité advienne à elle-même en s' incarnant à travers l'aperception affective inhérente à cette réciprocité sensible. Aussi, la subjectivité et le corps ne peuvent s'identifier ontologiquement qu'au terme de ce processus d'incarnation par lequel l'altérité, au sens spatial d'une réciprocité sensible, rend possible cet être. Le premier principe (et le premier enjeu) éthique réside, alors, dans l'exigence de reconnaître le statut accordé à cette altérité, en particulier dans l'aperception de l'homme par luimême.

I6. Il n'est pas question d'une décomposition de la pensée qui abstrait « le corps sans la sensation, le corps avant la sensation, c'est-ä-dire précisement l'acte de sentir considéré en lui-même et comme un pur pouvoir » (Henry, Philosophie, op. cit., p. Io8). Par l'antériorité du sentir sur les sens, j'entends la préorganisation des sens par ce sentir, au niveau sensible, et non leur exclusion d'une conception spirituelle de ce sentir où l'action, aperçue sous l'angle biranien du sentiment de l'effort, se transpose, curieusement, en « acte de sentir » afin d'identifier le corps et la subjectivité sous l'angle de la passivité.

17. L'empirie se définit par « toute intuition qui se rapporte à l'objet par le moyen de la sensation » (Kant, Critique de la raison pure, op.cit.. p. 79). 
l'aperception affective qu'il manifeste et qui concerne la totalité mise concrètement en œuvre, bien avant que le corps et l'outillage soient considérés indépendamment l'un de l'autre. Dès lors, l'outillage ne s'offre pas avant tout comme un espace distinct du corps, au sens d'un lieu l'environnant, mais comme partie prenante de la réciprocité sensible par laquelle l'aperception affective s'incarne en parvenant à ce corps, lors de l'action. Ainsi, dans le travail, mon action s'éprouve en intégrant l'outillage mis en branle et elle s'exerce en organisant, à la fois, l'outil et ce vers quoi il se porte, à travers les interventions corporelles que rendent possibles les différentes orientations de l'incarnation par lesquelles l'action s'effectue. Plus encore, si mon action fait partie de celle d'un groupe, je ne surviens à moi-même qu'au sein d'une tension existentielle mutuelle, par l'action de ce groupe, alors même que mon action contribue à y intégrer l'ensemble de l'outillage en question. En somme, « la réalité matérielle semble reculer à mesure que l'activité symbolique de l'homme progresse » (Cassirer, Essai sur l'homme, op. cit., p. 43) parce que, au fur et à mesure qu'il est question de l'aperception affective inhérente à l'action, le dynamisme de la sensibilité s'affirme et fait davantage sentir sa préorganisation sensorielle avant de libérer les sens pour que la réalité matérielle s'y forme empiriquement. Dès lors, l'homme ne se conçoit pas avant tout comme « animal symbolicum », comme l'exprime Cassirer (ibid., p. 44), mais comme affectivité symbolisante afin que le symbole, par lequel s'énonce le décentrement a priori de la raison, s'enracine dans ce par quoi il s'instaure.

Je reprends le cheminement de Kant et de Cassirer en les inscrivant dans le point de départ de cette affectivité symbolisante pour faire apparaître, à la fois, l'élargissement a priori du statut de la sensiblité que cela implique et l'intervention de ces deux problématiques dans la mienne, en particulier en ce qui concerne l'articulation spatio-temporelle. En apportant l'idée de l'espace comme réciprocité sensible, j'énonce le dynamisme spécifique de la sensibilité et je fais apparaître l'aperception affective inhérente à cette altérité que nos actions comportent de façon intrinsèque, comme nous l'avons vu précédemment. Je rends aussi possible l'aperception de cet espace en tant qu'il se temporalise lui-même, au sein de la réciprocité sensible, en changeant de statut afin de se définir par la référence au corps alors que sa forme a priori est, alors, dans l'esprit et non dans la sensibilité elle-mème, celle-ci étant devenue principalement empirique. Ainsi, Kant, dans sa propre problématique, ne prend pas en compte le processus dialectique ${ }^{\mathrm{I} 8}$ inhérent à sa façon d'instaurer l'espace et les

I8. Il me semble important de remarquer que l'esprit kantien produit dialectiquement 
difficultés qu'il a à commencer par l'espace. De fait, en ayant énoncé l'espace comme « extérieur à la chose » $(1768,90)$, il commence constamment par le temps tout en visant, seulement, l'espace qu'il tente d'établir en premier lieu. Par exemple, la Dissertation inaugurale commence par le temps puis enchaîne avec l'espace avant que la Critique de la raison pure puisse commencer par l'espace. Cependant, là aussi, il faut une seconde version pour remettre en question le privilège accordé au temps, dans la première version, à travers le statut accordé à l'imagination. L'absence du dynamisme spécifique de la sensibilité, chez Kant, se traduit par la formulation strictement rationnelle d'un dynamisme qui s'accentue dans la troisième analogie de l'expérience (l'action réciproque), en s'intègrant les deux premières (la substantialité et la causalité). Il s'agit d'une « communauté dynamique sans laquelle la communauté locale (communio spatii) ne pourrait être elle-même connue empiriquement » ( $178 \mathrm{I}, 240)$. Or, Kant prend appui sur la substance, en même temps qu'il permet d'apercevoir dans les trois principes synthétiques de la substantialité, de la causalité et de l'action réciproque, la condition intellectuelle pour formuler les trois rapports temporels possibles, en constituant la permanence, la succession et la simultanéité. En effet, c'est à la substance qu'il se réfère, peu après s'être référé au corps pour formuler dialectiquement l'espace au début de sa critique :

De même, si de votre concept empirique d'un objet quelconque, corporel ou non, vous retranchez toutes les propriétés que l'expérience vous enseigne, vous ne pouvez cependant lui enlever celle qui vous le fait concevoir comme une substance ou comme inhérent à une substance » (ibid., p. 6o).

Aussi, Kant formule l'action réciproque en s'appuyant sur la substantialité sans s'apercevoir que cette réciprocité se fonde dans

l'espace. En effet, l'esprit n'isole l'espace qu'en faisant abstraction de tout ce que la pensée y apporte par l'entendement avant de s'auto-régulariser lui-même rationnellement en accompagnant cette forme sensible qui « doit être a priori dans l'esprit » (ibid., p. 8o). Plus encore, Kant se réfère au corps pour le faire disparaître et en distinguer l'espace : « enlevez successivement de votre concept expérimental d'un corps tout ce qu'il contient d'empirique : la couleur, la dureté ou la mollesse. la pesanteur, l'impénétrabilité même, il reste toujours l'espace qu'occupait ce corps (maintenant tout à fait évanoui), et que vous ne pouvez faire disparaittre » (ibid. p. 6ol. L'esprit connaît par représentation à travers l'espace où le corps se dissout dans le phénomène. Ce que je propose, c'est, par exemple, l'aperception affective d'un espace latéralisé, dont la réciprocité sensible s'énonce là même où se trouve le corps auquel Kant se réfère. Elle permet l'incarnation en faisant surgir ce corps comme son terme avant d'en faire une référence initiale, dont l'espace se distingue, alors que la sensibilité est conçue empiriquement et que la raison s'impose. 
l'action elle-même, telle qu'elle s'énonce par la réciprocité sensible qu'elle comporte $a$ priori. De cette façon, à travers les analogies de l'expérience, il s'appuie sur la base du principe de la permanence de la substance, qui assure la formulation empirique des phénomènes dans le temps, et il transpose le changement, qui résume cette succession des phénomènes dans le temps, dans la loi de la liaison des effets et des causes afin de faire surgir la réciprocité (et, par là, l'espace) en l'ajoutant à l'action « comme si » elle était extérieure à celle-ci ${ }^{19}$.

Cassirer bénéficie de l'ensemble du parcours critique pour examiner de nouveau ces analogies de l'expérience et, en s'inspirant de la Critique de la faculté de juger, il leur donne un nouveau contexte : « il est indispensable à la pensée symbolique d'opérer une nette distinction entre le réel et le possible, l'actuel et l'idéal » (Essai sur l'homme, op. cit., p. 86). Là où Kant reste lié à la représentation des choses, par le schématisme, et où, dans la Critique de la faculté de juger, il conçoit le symbole comme un analogue du schème, Cassirer remet en question le rôle des images pour l'entendement humain et lui substitue celui des symboles. Il propose la symbolisation de la représentation des choses, de telle sorte qu'« un symbole n'a pas d'existence effective comme partie du monde physique; il a une "signification" » (ibid., p. 86). Au rapport kantien entre l'être et le devoir-être se substitue le rapport cassirien entre l'être et la signification, de telle sorte que la conception de l'homme ne s'appuie plus sur la substance mais sur la fonction, celle-ci s'exprimant à travers les formes symboliques. Ceci se traduit, au niveau de la culture, par une différenciation croissante entre les choses et les symboles, d' où l'insistance sur la distinction entre l'actualité et la possibilité. Tel est le point de départ de Cassirer ${ }^{20}$ pour complexifier le rapport des analogies de l'expérience avec les différents rapports temporels possibles : i) la conscience est exclusivement dominée par l'opposition de l'actuel et de l'inactuel, 2) certaines formes temporelles commencent à émerger par la séparation de l'action achevée (durable) et de l'action inachevee

19. Cet espace qui, finalement, se résume à une visée téléologique et s'appuie sur une conception substantialiste du temps se retrouve dans l'antithèse de la première antinomie tout en s'appuyant sur un même type de temporalité dans la thèse. $\mathrm{Ce}$ caractère tèléologique et le « comme si » par lequel il s'énonce se retrouvent dans la Critique de la faculté de juger, à travers ces productions de la nature qui surviennent « comme si elles étaient disposées tout exprès à l'intention de notre faculté de juger $\gg($ p. 320$)$.

20. Cassirer, Ia philosophie des formes symboliques, trad. O. Hansen-Love et J. Lacoste, Paris, Minuit, 1975, Tome I, p. 172. 
(momentanée) et à se différencier progressivement en modes temporels de l'action, 3 ) on parvient au concept pur de la relation temporelle, en tant que concept d'ordre abstrait faisant apparaitre clairement les divers degrés de temporalité dans leur opposition et leur limitation réciproques. Or, par l'animal symbolique, Cassirer privilégie le langage ${ }^{2 \mathrm{~T}}$ et se réfère au corps pour rendre compte de son élaboration de l'espace et du temps et, surtout, pour faire apparaître la différenciation et la symbolisation des rapports temporels comme étant plus complexes et plus difficiles que celles des rapports spatiaux. Cependant, le langage ne se formule pas seulement et avant tout selon un lien étroit avec la pensée symbolique, selon sa différence sentie par rapport aux choses, mais aussi selon son enracinement dans l'action elle-même. L'action met en oeuvre une réciprocité sensible et cette conscience de son altérité intrinsèque s'exprime comme une différenciation qui se sent elle-même en s'incarnant afin de pouvoir, aussi et par surcroît, transposer symboliquement sa spatialité, en la distinguant de ce corps, tout en énonçant la pensée elle-même de façon symbolique, en tant que cette symbolisation est considérée indépendamment du processus de cette transposition. Dès lors, le langage apparaît dans et par l'intervalle qui s'ouvre entre l'action et la pensée qui s'y génère et qui, par là, peut s'affirmer structuralement à l'encontre de cette action en s'appropriant le symbole pour parler d'elle-même, à travers la transposition indirecte du dynamisme sensible (l'affectivité qui l'anime) dans un dynamisme rationnel, par l'affirmation de la sensibilité empirique qui correspond à cette raison. D'où l'énoncé d'un « animal symbolique » mais aussi la possibilité d'une nouvelle percée de la métaphysique qui y est inscrite ${ }^{22}$.

21. La philosophie des formes symboliques débute par l'étude du langage. Cette conception langagière se généralise à l'ensemble de l'esprit, lors de l'étude non du mythe mais d'une « philosophie de la mythologie » (ibid., T.2, p. 15), et permet de formuler de véritables « actions de l'esprit » (ibid., T.2, p. 42) plutôt que des actions où s'enracine cet esprit, pour rendre compte du processus de division qui permet aux formes symboliques lle mythe, l'art, la connaissance...J de se séparer du réel immédiatement donné. A ces actions de l'esprit et à leur dissociation par rapport à l'action, succède l'étude des troubles pathologiques de l'action (et non de sa liberté, essentiellement subjective, par l'aperception affective) afin de dégager l'« esprit objectif $\gg$ (ibid., T.3. p. 311). Ce cheminement semble suivre celui décrit au sujet de la temporalité.

22. Cest ce que fait Heidegger en énonçant une « raison sensible pure » ou « imagination pure » (Être et temps, trad. E. Martineau, Paris, Authentica, 1985. p. 299) afin de mettre le Dasein au fondement de l'homme comme homme, de faire prévaloir le temps sur l'espace et de combattre la seconde édition de la critique de la 
Ainsi, je peux me dégager de toute pratique et clore métaphysiquement la différence entre l'outillage et l'outil sur elle-même en l'énonçant spéculativement par une différence ontologique que je formule indépendamment de moi, selon une pure métaphysique ${ }^{23}$. Je peux aussi la penser en lui prêtant ma parole afin d'affirmer que c'est par le Dasein que se déploie le fait lui-même d'une différence ontologique, de telle sorte que ce Dasein doit découvrir son authenticite au lieu de se perdre de façon inauthentique dans ce monde habituel mis en jeu par une pratique. Cette pratique est inauthentique lorsqu'elle est considérée indépendamment de l'angoisse ${ }^{24}$ inscrite dans le langage, ce

raison pure qui « se décide pour l'entendement pur et contre l'imagination pure afin de sauvegarder la suprématie de la raison » (ibid., p. 225). Or, les deux problématiques, celle de Cassirer et celle de Heidegger, puisent au même privilège accordé à la formulation empirique plutôt qu'à la formulation affective de la sensibilité, bien que Cassirer s'y maintient pour affirmer la pensée symbolique et que Heidegger s'en écarte a priori, par l'imagination, pour affirmer la « métaphysique du Dasein » (ibid., p. 286), là même où pourrait s'annoncer une théorie de la connaissance et l'introduction à la pratique que celle-ci est supposée instaurée, chez Kant.

23. La pratique réelle inhérente à l'action, en se transposant en une pratique close sur elle-même devient technique, par la pure différenciation entre l'outillage et l'outil qui s'impose à l'homme, et développe une universalité à l'encontre de la singularité de cet homme. En effet, l'idée de l'indépendance de la nature apparaît à travers l'idée de l'indépendance de la différenciation entre l'outillage et l'outil mais en oubliant la genèse de cette idée à partir de l'action. De même, l'idée d'une dialectique purement spéculative prétend reconstituer une liberté humaine en partant de la pure extérioritê des termes entre l'homme et la nature. Ainsi, la pratique close sur elle-même peut être un moment de la pratique réelle, inhérente à l'action, et ou peut être assimilee à ce moment et s'absolutiser, comme chez Hegel ( 1807 ), dans la recherche d'un savoir se développant à l'encontre de l'homme, tel qu'il s'apparaît à lui-même à travers ses actions. L'immanence propre à la singularité agissante se trouve alors transposee dans celle du concept, mais elle peut aussi être réduite à cette singularité, en étant identifiée au corps, comme chez Maine de Biran.

24. Heidegger écrit : « Certes, il appartient à l'essence de toute affection d'ouvrir à chaque fois l'être-au-monde plein selon tous ses moments constitutifs (monde, être-là, Soi-même). Néanmoins, s'il y a dans l'angoisse la possibilité d'un ouvrir privilégiè, c'est parce que l'angoisse isole. Cet isolement ramene le Dasein de son échéance et lui rend l'authenticité et l'inauthenticité manifestes en tant que possibilités de son être » (ibid., p. I47). La distinction entre l'angoisse et la peurpermet d'apercevoir la différence heideggérienne entre l'être et l'étant à laquelle s'ouvie la distinction entre l'authenticité et l'inauthenticité du Dasein : « La peur a son occasion dans l'étant offert dans le monde ambiant à la préoccupation. L'angoisse, au contraire, jaillit du Dasein même. La peur assaille à partir de l'intramondain. L'angoisse s'éléve à partir de l'être-au-monde comme être pour la mort jeté » (ibid., p. 241). À cette distinction répond celle entre l'affectivité inherente à l'action, que je propose, et l'affectivité spirituelle, proposée par Henry, afin d'apercevoir la différence entre l'altérité, impliquée par l'action, et l'être, impliqué par l'antinomie de ses métaphysiques. 
langage s'adressant à l'être pour que l'être puisse se dire lui-même, littéralement, en rendant compte de cette pratique ${ }^{25}$. Enfin, je peux faire apparaître, comme chez Henry, le corps par lequel s'énonce métaphysiquement l'autre terme avec lequel la pensée affirme son dualisme jusque dans le rejet lui-même de ce dualisme, quoique ce soit en faisant apparaître le rapport de réversibilité entre l'intentionnalité mise en jeu par la pensée et la singularité mise en jeu par le corps. L'affectivité se sépare, alors et alors seulement, de la totalité symbolique mise en jeu par l'action et s'identifie au corps, de telle sorte que le sentiment de soi puisse y être rapporté comme à un sentiment du Soi et que les sens puissent se dégager de leur préorganisation affective, tout en étant sus ceptibles d'être exclus de la conception de l'affectivité, au sens spirituel que lui donne Henry.

Deuxièmement, la définition de l'affectivité implique un rétablissement de la sensibilité dans l'aperception que l'homme a de lui-même et non une nouvelle définition de l'esprit s'appropriant le concept à sa façon tout en contraignant l'esprit au sens intelligible. Dès lors, en faisant intervenir le symbole plutôt que la raison kantienne proprement dite qui fait du symbole un analogue du schème ${ }^{26}$, il est possible de transformer le statut de l'intelligiblité au niveau de cette intelligibilité elle-même, comme le propose Cassirer. En effet, la problématique de Cassirer, en ce qui concerne l'idée même des formes symboliques, s'enracine dans La critique de la faculté de juger. Aussi, la distinction kantienne entre le réel (au sens de l'effectivité) et le possible permet d'introduire la différence entre les

25. Il me semble important de préciser ce lien avec la pratique, qui se trouve « allégée » et décalée au profit de l'intégration réflexive des questions de la pensée et de l'être dans le langage, lorsque nous cherchons à montrer « comment l'ontologie, d'un langage sur l'être devient nécessairement le langage de l'être où l'être lui-même se dit dans une auto-logie sans fissure » (Yvon Gauthier, Théorétiques. Pour une philosophie constructiviste des sciences, Longueuil, Le Préambule, 1982, p. 224, note 3). C'est du point de vue de l'action qu'il est question d'un tel lien « allégé » et décalé par rapport à la pratique, puisque, du point de vue du langage, qui formule ce lien, la rupture est premiére du fait de la spécificité de ce langage. Cependant, même si l'on accepte, à l'encontre de Heidegger, une scission entre le langage et l'être, il reste encore la question du lien entre le langage et la pensée. Or, dans ce cas, il y a un nouveau choix entre le langage qui survient dans l'intervalle entre l'action et la pensée (en tant qu'elle parvient à l'existence) et le langage par lequel la pensée se dit elle-même en une expression sensible distincte de celle de cette action, du fait que cette expression sensible est avant tout empirique et que son contexte s'énonce comme une structure analysable (et non plus générée).

26. Kant, Critique de la faculté de juger, trad. A. J.-L. Delamarre, J.-R. Ladmiral, Marc B. de Launay, et J.M. Vaysse, introd. et dir. F. Alquié, Paris, Gallimard, La Pléiade, $198_{5}$, p. 314 . 
choses et le libre « "espace symbolique" de la représentation » (Cassirer, Philosophie des formes symboliques, op. cit., T.3. p. 305) de ces choses. Cette distinction synthétise la critique kantienne et en dégage la réflexivité puisque le trajet de La critique de la raison pure, permettant de cerner ce qu'on entend par « réel », ne conduit à La critique de la raison pratique, faisant état de ce qui est « possible » par la liberté, que pour revenir du possible au réel, c'est-à-dire à « la mise en place de la chose en soi-même (en dehors de ce concept) » (Kant, Critique de la faculté de juger, op. cit., p. 371). Or, tout comme Kant faisait place à la pratique dès sa critique de la raison théorique, il est possible de faire place, dès ce trajet de la théorie vers la pratique, au retour réflexif de cette pratique sur la théorie au lieu d'en faire une pure succession chronologique et de traiter, depuis ce point de vue chronologique, le second terme (le retour réflexif) comme un analogue du premier (le trajet initial de la théorie vers la pratique). Cassirer, en dégageant la spécificité des formes symboliques nous permet d'adopter le nouveau point de vue de ce second terme mais il ne nous permet pas d'apercevoir ce que cela implique comme transformation initiale du premier terme. C'est ce que j'apporte par l'affectivité qui préorganise les sens, avant toute formulation purement empirique de ces sens, celle-ci pouvant sous-tendre la distinction logique entre l'entendement et la raison lors de la formulation des formes a priori de la sensibilité. En même temps, je propose une nouvelle approche de la liberté, à travers la définition du symbole par l'aperception duale, à la fois affective et rationnelle, de l'homme, où la raison n'apparaît qu'à travers son decentrement a priori qu'implique, en l'homme, son affectivité. Il en résulte non plus une « culture objective » (Cassirer. supra, T.3, p. 3II) mais bien une culture subjective, au sens d'une culture s'éprouvant elle-même. Dès lors, contrairement à Henry, je n'abstrais pas l'affectivité de la sensibilité, ce qui me permet de reconnaître son hétérogenéité par rapport à la raison, et je transforme, comme Kant et Cassirer, l'intelligibilité elle-même pour introduire une nouvelle conception de l'esprit, au lieu de faire de cette affectivité une nouvelle conception de l'esprit ${ }^{27}$.

Cette opération est l'inverse de celle de Henry qui s'adresse à l'affectivité pour y apercevoir cette nouvelle conception de l'esprit, celle-ci étant issue du fait de la contrainte du corps sur la pensée. Ainsi, il est possible de s'interroger à la fois sur la différenciation de la

27. La difficulté essentielle de ma démarche réside dans le fait que, en faisant une place centrale à la sensibilité dans la conception de l'homme. c'est tout l'homme qui se transforme, son esprit inclus, et non seulement cet esprit. 
sensibilité en affectivité et en sensorialité, sur l'étroitesse de la conception de l'affectivité comme conception de l'esprit, en tant qu'elle reconduit le rationalisme au sein même de la sensibilité au détriment de celle-ci et, enfin, sur la spécificité de la raison elle-même, telle qu'elle se distingue de l'entendement par lequel s'organise la perception sans que, pourtant, l'objet de cette dernière se réduise directement à la seule sensation comme semble le croire Henry, dans la mesure où il combat réellement la philosophie de l'intentionnalité ${ }^{28}$. Cette suppression de

28. Deux sous-problèmes ètroitement liés apparaissent ici, celui de la circularité lieee aux prémisses du discours de Henry et celui de l'orientation donnée aux sens pour, à la fois, s'adresser à Heiddeger en le combattant et évacuer toute conception de l'affectivité qui pournait s'orienter sur une voie d'inspiration kantienne. L'analyse de la circularité, inhérente à la réversibilité des différents types de métaphysiques qu'accumulent Henry, fait apparaître que Henry déplace implicitement la question de l'intentionnalité au niveau des sens, en deça de la perception que l'entendement organise pour qu'il y ait une représentation au sens empirique, et/ou il refuse explicitement l'intentionnalité en tant qu'elle relève de cette perception pour avoir lieu comme connaissance, ce qui peut lui permettre d'empêcher l'aperception du caractère problématique de cette intentionnalité implicite qui ne peut pas en être vraiment une ou qui, du moins, pourrait se prêter au discours en s'explicitant. Ainsi, Henry commence par affirmer que « le problème de la réceptivité de la connaissance à l'égard de l'étant est celui de sa réceptivité à l'égard de l'objet puisque c'est justement comme objet que l'étant est reçu » (L'essence, op.cit., p. 156). Ultérieurement, il précise : « En tant que la sensation se réfère à un objet dont elle est la sensation, elle est représentative. Qu'elle « représente » un tel objet ne signifie nullement qu'elle le pense, qu'elle se le représente dans un acte de connaissance, que cet objet soit l'objet déterminable de l'entendement » (ibid., p. 628). Aussi, de l'affectivité a la perception, il y a « une relation inverse », comme une diminution progressive de l'affectivité tandis que se développe au contraire la représentation elle-même, la sensation représentative ou sensation proprement dite » (ibid., p. 631). Or, la sensibilité affective, dont il est question ici, se lie au corps en s'explicitant conceptuellement comme une nouvelle conception de l'esprit, par la contrainte de ce corps sur la pensée, ce qui permet de définir cette affectivité. En même temps, ce qui surgit de la sensation, c'est un monde par lequel Henry peut, à la fois, rendre possible son opposition à Heidegger, en affirmant que le néant où survient le monde ne peut s'effectuer que par l'affectivité, et purifier le point de vue de Maine de Biran qui, au lieu d'un monde, fait intervenir la connaissance, dont celle du moi, qui ne pourrait commencer « qu'à l'exercice d'une activité hypersensible et hyperorganique, c'èst-à-dire au premier acte de vouloir, à l'effort ou encore au mouvement non pas senti, mais voulu et opéré par une force qui est en dehors de la sensation et supérieure à elle » (Maine de Biran, CEuvres choisies, op. cit., p. 6g). Finalement, Henry, en faisant surgir la représentation du rapport de la sensation à l'objet, ne peut évacuer la problématique kantienne que dans la mesure où il refuse aussi le caractère empirique de la perception que cela implique, ce qui lui permet de faire intervenir cette transcendance du monde à laquelle il peut s'opposer au lieu de renvoyer cette perception à l'entendement et aux formes a priori de la sensibilité. En effet, chez Kant, c'est par ces formes a priori de la sensibilité que l'esprit peut accèder au caractère empirique de cette perception, pour rendre compte de la nature (et non seulement du monde), en tant que cet esprit s'en distingue comme liberté pratique. 
la distance de la perception par rapport à la sensation, pour établir la référence à l'objet, n'est pas accidentelle, puisque cette distance est nécessaire pour énoncer le doute cartésien et le fait de son exercice par l'entendement ainsi que la formulation de la représentation par laquelle s'instaure la philosophie de l'intentionnalité. De plus, il y a différentes façons de remettre en question cette intentionnalité. Il est possible de suivre Heidegger, comme le propose Henry, en faisant reposer l'espace dans le monde et en affirmant que « le concept de distance phénoménologique n'est point liéa l'espace » (L'essence, op. cit., p. 76). Cependant, il se pourrait que cette distance phénoménologique soit, au contraire, avant tout spatiale, si l'on veut bien concevoir l'espace comme une réciprocité sensible au sein de laquelle le monde ne peut surgir que de façon symbolique, par et dans l'action, avant de donner lieu à un tel renversement du rapport entre le monde et l'espace où la question de l'intentionnalité se donne sans sa condition symbolique de possibilité. Dans ce cas, la critique de la représentation ne peut signifier le rejet de cette représentation hors de l'affectivité, au sens spirituel que lui donne Henry, que par une critique la précèdant; celle-ci fait apparaître le contexte strictement rationnel de cette représentation afin de faire place à l'affectivité, au sens sensible, dans l'aperception. Ainsi, la « distance » s'intériorise dans l'aperception elle-même et se définit par le caractère sensible de l'altérité, qui s'énonce par l'affectivité, bien avant de passer par l'être qui se trouve lié à la raison. La réciprocité sensible donne alors accès, quoique indirectement, au corps, au monde et à leur rapport spirituel. Au langage de l'intentionnalité répond, alors, le langage articulant le rapport entre le sensible et l'intelligible selon différentes orientations, l'affectivité et la raison, tout en instaurant une polarisation des termes du discours, du fait que l'affectivité s'énonce par la symbolisation (le décentrement a priori de la raison) et que la raison s'exprime en se référant d'emblée aux sens, que ce soit pour s'y appuyer (par la distinction entre l'entendement et la raison), s'en écarter (par l'imagination) ou, encore, les exclure (par l'affectivité de la pensée). En somme, le lien entre le langage et la pensée se relativise en même temps qu'apparaît le lien entre le langage et l'action auquel il nous introduit. L'action, en s'articulant, permet à l'articulation du langage de s'énoncer en tenant compte du contexte intersubjectif par lequel elle se produit.

Troisièmement, c'est l'action qui s'éprouve elle-même comme réciprocité sensible jusque dans la totalité symbolique qu'elle met en oeuvre par la mise en tension de tout ce qu'elle intėgre pour s'effectuer. $\mathrm{Au}$ sein de cette action, le corps ne peut s'énoncer comme la subjectivité elle-même que dans la mesure où cette subjectivité déserte la totalité symbolique mise en oeuvre, tout en faisant peser un monde sur 
elle-même, et où elle réinterprète la réciprocité sensible (et l'espace luimême) à travers un tel corps subjectif afin d'en faire un être-opposé et de se la donner pour la subir au sein d'une action identifiée à l'effort. Ce qui est donné à l'effort, « c'est la tension intérieure de l'existence qui affronte l'être-opposé et dans cet affrontement se le donne, c'est l'effort, mais dans la façon dont leffort est donné à lui-même, dans le sentiment de l'effort, il n'y a pas d'effort » (Henry, L'essence, op. cit., p. 595). Ici, « subjectivité » ne veut plus dire « agir », « altérité », mais « veut dire «subir », veut dire « être » (Henry, ibid.). Aussi, ce qui m'est absurde s'énonce à travers mon action et m'apparaît en son hétérogénéité : je présuppose que l'action présuppose l'affectivité et que l'affectivité s'éprouve en soi, indépendamment du contexte originaire de l'action par lequel s'énonce le sentiment de soi qui lui est inhérent. Avec Henry, je m'emprisonne dans l'identification du sujet à l'effort musculaire, même s'il m'amène à m'écarter de celle-ci pour n'en retenir que l'idée du sentiment propre à cette subjectivité et pour énoncer la théorie ontologique de l'affectivité. Tout comme la perception s'est effondree dans la sensation, la pratique, au sens moral, s'effondre dans la pratique, au sens technique, tel qu'il peut être rattachée au sentiment d'effort, alors qu'un monde pèse sur le corps subjectif. Ainsi, l'affectivité ne serait « la condition de la moralité que parce qu'elle est en général celle de l'action » (Henry, ibid., p. 662). A partir de cette dichotomie entre l'action identifiée à l'effort et l'« autoaffection de l'acte qui pose la loi, dans l'affectivité de la raison elle-même en tant que pratique » (Henry, ibid., p. 666), Henry peut écrire : « La substitution du respect à l'amour a une signification ontologique ultime, elle est la substitution de la structure de l'affection pure à celle de l'affectivité » (ibid., p. 666). L'articulation ontologique qui s'énonce, ici, découle de la conception ontologique de l'affectivité que propose Henry et méconnaît le fait que le problème qui se pose, chez Kant, se situe au niveau d'une articulation méthodologique qui est énoncée par la critique de la raison, dont je propose de reconsidérer l'aperception rationnelle pour faire place à une aperception duale, à la fois affective et rationnelle, de l'homme. En effet, chez Kant la substitution du respect à l'amour n'est pas « la substitution de la structure de l'affection pure à celle de l'affectivité » (Henry, ibid., p. 666). C'est Henry qui, après avoir formulé l'affection au sens de l'étant reçu, la substitue à l'empirie sous-tendant la raison kantienne et dissocie l'affectivité de la sensibilité pour substituer une conception de l'esprit à cette sensibilité, là où Kant la reconnaît mais en la transposant dans le respect. Il s'agit de la seule façon par laquelle Kant pouvait rendre compte de l'affectivité du point de vue de la raison alors même qu'il en reconnaissait l'hétérogénéité sensible, mais étroitement liée à ses sources empiriques, par 
rapport à la raison. Reconnue comme quelque chose mais n'ayant pas de nom propre, elle ne recevait d'autre nom que celui que la raison pouvait bien lui donner, tout en étant affectée par elle ${ }^{29}$. Cependant, la position kantienne recèle la possibilité du symbole et, par là, principalement d'un dire, selon Cassirer, et d'une action, selon la présente thèse, qui permet, dans ce dernier cas, de reconnaitre la spécificité de l'affectivité. Il s'agit, par la symbolisation, de restituer l'antériorité spécifique de l'affectivité par rapport à ce qu'en dit la raison, en l'homme, ce qui ne signifie pas une antériorité absolue mais bien une restitution de celle-ci dans la mesure où l'homme parvient à dénouer le cercle rationaliste où il s'enferme et, cela, par la symbolisation qui l'amène à se reconnaître luimême par une aperception duale.

Aussi, Henry a tort d'apercevoir dans « l'autoaffection de l'acte qui pose la loi » (Henry, ibid., p. 666), un accès direct à l'aperception immédiate qui serait inhérente à une affectivité issue de la contrainte du corps sur la pensée. La totalité symbolique, propre au contexte de l'action, fait apparaître que s'il y a une aperception immédiate inhérente à l'affectivité, celle-ci survient indirectement, au sens spatial du terme, par l'altérité inhérente à la réciprocité sensible par laquelle elle s'incarne. C'est par cette altérité que l'action s'aperçoit affectivement et qu'elle permet, à travers le symbole, de rendre compte de « l'être de l'action $»^{30}$ ainsi que de sa réduction « au pathos de l'effort au sein de la subjectivité individuelle » (ibidem). Ainsi, la réciprocité sensible, comme espace latéralisé, permet l'accès du corps à lui-même dans la mesure où l'aperception rationaliste de la différence subjective entre les deux côtés du corps ${ }^{3 \mathrm{I}}$ s'enracine dans l'aperception affective de l'équilibre symbolique, entre les différentes orientations gyratoires. Au sein

29. " $S$ 'il y a lieu d'instituer quelque "critique de la raison pure", c'est que l'homme se définit bien par la raison, en toute sa force (en sa "pureté"), mais qu'elle ne se rencontre jamais en lui que sous la figure d'une "raison affectée" » (F. Marty, « Raison pure et raison affectée. Å propos de l'affectivité chez Kant » dans R. Legros, P. Loraux et M. Richir (dir.), Pensée et affectivité, Jérôme Millon, I99ı, p. g). Or, ceci s'affirme en même temps que le terme d'affectivité « est absent de l'oeuvre publiée du vivant de Kant » (idem). Dés la Critique de la raison pure, Kant a « en vue un objet étranger à la philosophie transcendantale » (op. cit., p. 600) et précise, dans une note, le lien entre les concepts pratiques et le sentiment, en tant qu'il réside en dehors de toute faculté de connaître.

3o. Serge Cantin, « Grandeur et limite du Marx de Michel Henry », Dialogue, vol. XXIX. N 3. p. 395.

31. Kant « Du premier fondement de la différence des régions dans l'espace », dans Quelques opuscules pré-critiques, Paris, Vrin, 1970, p. 93-98; et Kant, Qu'est ce que s'orien ter dans la pensée?, trad. et commentaire A. Philonenko, Paris, Vrin, 1972, p. 75-8g), 
de cette aperception affective, chaque côté du corps n'apparaît qu'à travers la tension inhérente à la double façon de produire la différence subjective, puisque la relation entre les deux côtés du corps s'énonce par une tendance posturale, à la fois préférentielle et universelle, vers la droite qui est plus ou moins équilibrée par une tendance vers la gauche. Je n'accède à mon corps que par l'équilibre dynamique et différencié de ma latéralité dont l'organisation spatiale me permet de m'incarner par l'aperception affective de l'enchaînement de ses moments. De fait, je ne m'incarne subjectivement par cet espace latéralisé qu'en permettant l'incarnation de l'altérité elle-même, comme dans le rapport intersubjectif impliquant un outillage ${ }^{32}$. Aussi, la réciprocité sensible inhérente à l'action, par l'articulation du rapport intersubjectif à l'espace latéralisé, permet l'accès au processus de l'incarnation, au

ici aussi, a aperçu l'importance de la latéralité même s'il ne la nomme pas comme telle. Il a même aperçu le lien entre la latéralité et l'orientation dans l'espace : «S'orienter signifie en son sens propre : trouver à partir d'une région céleste donnée - nous distinguons l'horizon en quatre régions - les autres régions et surtout l'orient. Si je vois le soleil au ciel et si je sais qu'il est maintenant midi, je puis trouver le sud, l'est, le nord et l'ouest. Mais à cet effet il m'est indispensable d'éprouver par rapport à moi-même le sentiment d'une différence, je veux dire celle de la droite et de la gauche » (ibid., p. 77). Cependant, Kant n'a pas aperçu la latéralité comme un espace là même où se trouve le corps et il n'a pas envisager son caractère dynamique, bien que la différence subjective entre la droite et la gauche se double aussi, « malgré leur ressemblance extérieure » (« Du premier fondement », op. cit., p. 95), d'une différence entre la force du mouvement (qui semble davantage liée au côté droit du corps) et la prépondérance de la sensibilité (du côté gauche). Il m’apparait important de remarquer la contemporanéité du rationalisme kantien et de l'énonciation de l'espace comme « extérieur à la chose » (ibid., p. go), qui se trouve à la base de la decouverte de sa philosophie.

32. Un second principe éthique réside dans cette exigence de permettre à l'altérité de s'incarner afin de pouvoir s'incarner soi-même. Il est solidaire du premier qui énonce l'exigence de reconnaître le statut accordé à l'altérité au sein de la conception de l'homme. De fait, il s'agit d'un unique principe éthique dont je donne maintenant une expression synthétique, au sens symbolique, après en avoir donné une expression analytique (la reconnaissance du dynamisme de l'altérité, qui s'affirme par celui de l'affectivité, impliquant le rétablissement a priori de la sensibilité dans l'aperception). Il est important de remarquer que cet ordre d'exposition se déroule à l'inverse de l'ordre réel, qui est inhèrent à l'action, et que sa fonction est, avant tout, d'y introduire. La symbolisation se définit pratiquement par cette antériorité à laquelle nous accédons après coup, par le point de vue spéculatif où la synthèse existentielle se transpose rationnellement dans une synthèse qui est seconde par rapport à l'analyse. Ainsi, le rapport éthique ne se rend possible au sein même de la conception de l'homme, par la dualité de l'aperception, que pour s'exprimer concrètement à travers notre action, dont l'horizon symbolique permet l'articulation de cette aperception duale. 
terme duquel le corps surgit ${ }^{33}$, en introduisant le rapport rationnel qu'il entretient avec la pensée et en faisant surgir spéculativement une totalité (davantage logique que symbolique) dégagée de l'action. Dès lors, l'affectivité symbolisante ne se réduit pas à « l'affectivité de la raison elle-même en tant que pratique » par laquelle Henry (L'essence, op. cit., p. 666) critique Kant, puisque le statut accordé à cette raison pratique

33. Le corps surgit en faisant, aussi, apparaitre un espace distinct de lui. cet espace l'environnant par l'intègration de l'apparence des tendances gyratoires s'y déroulant à l'inverse de celles de l'espace latéralisé. De plus, l'idée de l'altérité, qui s'annonce par un tel espace distinct du corps, s'affirme elle-même, au niveau de la pensée, par la distinction entre cette idée et l'altérité réelle, telle qu'elle s'aperçoit intersubjectivement afin d'introduire a priori une autre subjectivité en regard de la mienne et d'y envisager la tension (si elle est maintenue) entre l'incarnation et le corps qui surgit au terme de celle-ci. Enfin, l'outillage, en se dégageant de l'action alors que cette action s'y oublie, peut apporter l'idée de l'indépendance à l'idée de l'espace environnant, qui apparaît, maintenant, là où la latéralité lui apporte l'idée de son apparence. De l'apparence à l'apparaitre puis à l'affirmation de ce qui apparaît, par la pensée établie à distance de l'action, la nature reçoit son nom en regard de son fondement corporel, au fur et à mesure que cette pensée s'énonce structuralement en s'analysant spéculativement (et non plus en se générant pratiquement dans l'action qui exige, a priori, des limites concrètes à ces structures). Dès lors, l'outillage explicite sa matérialité à travers celle d'une natureen se thématisant conceptuellement par un espace formel devant être a priori dans l'esprit. Ia formulation dialectique de l'espace, sur le fondement du corps, produit le schématisme de la substance dans la permanence du phenomène comme apparence; sa formulation indépendante produit le schématisme de la cause dans la successivité du phenomène comme apparaitre, sa formulation matérielle produit le schèmatisme de l'action réciproque dans la simultanéité du phénomène comme concept de ce qui apparaît par cet apparaître. En somme, l'action s'éprouve par sa réciprocité sensible et celle-ci s'incarne en conduisant au corps qui sous-tend le développement du schématisme de l'entendement, tel qu'il s'adresse à la nature. Le caractère pratique de l'action est, donc, la condition de possibilitè du caractère spéculatif de la pensée, cette pensée s'apercevant naturellement comme nature, c'est-à-dire en objectivant sa subjectivité. Aussi, la nature humaine, postulée comme et par l'expression rationnelle de la pensée, régularise la formulation de la nature par le schèmatisme de l'entendement. Cette régulation, cependant, tient dans la vérité que comporte l'écologie : la nature est une introduction à soi-même, dans la mesure où, par le point de vue speculatif qui s'impose par elle, c'est notre point de vue pratique qui, déjà, se manifeste. Par conséquent, la nature absolue est l'inconscience absolue, puisque l'imposition spéculative s'assimile alors la pratique au lieu d'y introduire, de telle sorte que la conscience disparait dans la pensée et que le langage se lie exclusivement à la pensée en se dégageant de toute action, sauf si la réalité de cette action se trouve déplacée et réduite à une expression technique par laquelle, littéralement, elle se schematise (de façon principalement et initialement spatiale et non seulement de façon principalement temporelle et téléologiquement spatiale, comme chez Kant). 
n'ouvre pas tant la voie à l'inflexion rationnelle de la pensée par le corps qu'à la voie du symbole, tel qu'il s'enracine dans l'altérité inhérente à la réciprocité sensible par laquelle l'action s'aperçoit affectivement en s'effectuant.

En ce sens, il apparait important de s'interroger sur le rapport entre la liberté, que formule Kant, et la non-liberté, par laquelle « l'affectivité détermine l'action et la précède comme son essence » (Henry, L'essence, op.cit., p. 823). Ici se pose la question qui résume le débat : quel rapport ma liberté entretient-elle avec ma non-liberté et comment peut-elle s'annoncer dans le rapport kantien de la liberté à la nature ? La réponse surgit du développement précédent : l'action implique une altérité, au sens spatial d'une réciprocité sensible qui s'éprouve et s'énonce symboliquement, de telle sorte que le rapport de ma liberté à ma non-liberté s'énonce par l'ouverture a priori de la raison à l'affectivité. L'altérité de l'affectivité, au sein de l'aperception, fait apparaître la raison, depuis cette affectivité, selon un décentrement a priori. L'homme symbolise et cette symbolisation permet de maintenir la tension entre le sensible et l'intelligible par l'aperception duale, à la fois affective et rationnelle, au lieu de supprimer cette tension et de faire de l'affectivité une nouvelle conception de l'esprit.

Cependant, cette symbolisation ne peut se dégager de la problématique kantienne qu'en dénouant le paradoxe inhérent au sentiment moral, ce sentiment qui n'en est pas un et / ou qui en est un en écartant tout sentiment, et en faisant intervenir l'affectivité, comme dynamisme spécifique de la sensibilité, dès la formulation empirique de la sensibilité qui sous-tend la distinction entre l'entendement et la raison. Il s'agit de s'intéresser à la façon par laquelle le respect est un sentiment moral, le seul, plutôt que d'affirmer que « l'affectivité du respect est laissée de côté, seule est retenue en lui la relation à la loi, la transcendance » (Henry, ibid., p. 665). En effet, c'est le paradoxe du « sentiment moral » (Kant, Critique de la raison pratique, op. cit., p. iog) qui permet d'énoncer le rapport kantien de la conscience de la loi morale aux inclinations par l'humiliation (le mépris intellectuel pour les inclinations) et le respect (pour la loi morale). Ce paradoxe fait apparaître l'affectivité non comme une altérité dans l'aperception mais comme un infini qui se substitue à cette altérité puisque cette affectivité éclate entre son néant et sa reprise infinie en termes rationnels par la pensée. Or, c'est la raison elle-même qui se piège ainsi, puisque ce qui devait être « une coercition inévitable exercée sur toutes nos inclinations, mais seulement par notre propre raison » (Kant, ibid., p. 115), apparaît comme une contrainte de l'affectivité sur cette raison, du fait que «dans l'affect la liberté de l'esprit est certes entravée, mais elle est 
supprimée dans la passion » (Kant, Critique de la faculté de juger, op. cit., p. 217 , note $)^{34}$.

Nous aurions, ici, un cas où Kant a entravé et supprimé sa propre démarche critique et ceci parce qu'il en a fait une critique de la seule raison ${ }^{35}$, de telle sorte que nous pourrions dire avec Kant lui-même : « on s'aperçoit bientôt que là où l'entendement ne peut suivre, la raison devient excessive » (ibid., p. 37o). L'entendement ne peut suivre parce qu'il n'a de pouvoir que sur les perceptions et qu'il ne peut savoir ni quand, ni où intervenir tant que les sens n'ont pas été dégagés de leur préorganisation affective. C'est pour la raison qu'il est question d'une empirie disponible au schématisme de l'entendement et d'une nature par rapport à laquelle se définit sa liberté. Tant qu'il y a confusion entre les sens, tels qu'ils sont préorganisés affectivement, et les sens, tels qu'ils sont rendus disponibles à la différenciation logique entre l'entendement et la raison, l'entendement risque de multiplier les événements qu'il ne peut suivre et la raison se noue elle-même dans un tissu paradoxal qui, au lieu de la stimuler, l'emprisonne dans l'aporie. Il faut, donc, élargir le statut accordé à la sensibilité si nous voulons nous rendre accessible le passage entre l'affectivité et la perception. Il s'agit d'un point de vue méthodologique sur ce passage, par lequel il s'agit de préserver l'altérité essentiellement sensible dont il est question ainsi que sa différenciation et son dynamisme. Il ne s'agit pas, en premier lieu, d'une affirmation ontologique qui présuppose ce passage avant même de rendre compte de son accès à l'altérité énoncée par la sensibilité, telle qu'elle est en question dans ce passage et telle qu'elle nous engage au sein de nous-mêmes, comme homme et non seulement comme esprit. C'est ce rapport complexe entre le sensible et l'intelligible, par lequel s'énonce déjà la liberté kantienne, qu'il s'agit de préserver même si, dans une nouvelle problématique, le symbole s'énonce par un

34. Le processus (et non seulement le produit) de cette contrainte de l'affectivité sur la raison est mis en évidence dans cette citation; « Dans l'èmotion, l'esprit surpris раг l'impression perd l'empire de soi-même » (Kant, Anthropologie du point de vue pragmatique, trad. M. Foucault. Paris, Vrin, 1964, p. Iog). Du fait de l'absence d'une ouverture a priori de la raison à l'affectivité, le confinement de l'affectivité aux sources empiriques de la sensibilité, que la raison présuppose alors, conduit cette raison à être surprise et, par là, à être entravée, c'est-à-dire à perdre l'empire d'ellemême. Ultimement (et cela échappe à une telle raison). c'est l'homme lui-même qui est entravé en son humanité, puisqu'il ne peut pas s'apercevoir affectivement de façon a priori, lors de cette stricte identification à la raison.

35. Ceci se traduit, en particulier, par le sublime, c'est-à-dire par l'assimilation de l'affectivité au « sentiment que nous possédons une raison pure autonome » (Critique de la faculté de juger, op. cit., p. 200). 
décentrement a priori de la raison et si la sensibilité se définit de façon dynamique par l'affectivité avant de se définir par la préséance que la raison accorde à l'empirie. En introduisant l'affectivité comme dynamisme de la sensibilité là où Kant (Critique de la raison pure) propose un dynamisme rationnel portant sur les formes a priori de la sensibilité empirique $^{36}$ et en reconnaissant cette spécificité de la sensibilité là où

36. Il serait risqué de dire, au sens strict, que Kant identifie la sensibilité aux sens, étant donné les formes a priori de la sensibilité. Cependant, il n'est pas possible d'ignorer que le dynamisme de la raison ne donne lieu aux formes a priori de la sensibilité qu'en privilégiant, au sein de la sensibilité, la dimension empirique de celle-ci. Si l'on ne tient pas compte de la possibilité d'èlargir le statut de la sensibilité, à laquelle Kant fait déjà une grande place, comment distinguer, par exemple. dans ce qu'on énonce à travers le dynamisme de la raison, ce qui appartient en propre à cette raison et ce qui ne serait qu'une transposition du dynamisme spécifique de la sensibilité mais que l'on prendrait pour un dynamisme rationnel, comme dans le cas du paradoxe où le sentiment moral risque de nous entraîner ? Comment rendre compte de la démarche kantienne elle-même, qui propose le sujet plutôt que l'objet comme centre de notre réflexion, en ce qui concerne la connaissance, mais en occultant cette subjectivité elle-même quant à son aperception affective afin de l'ordonner à une visée téléologique l'objectivant, à travers la perception par laquelle la symbolisation et, par elle, l'homme lui-même se trouvent réduits à la seule aperception rationnelle qui s'y rend possible? De même, chez Cassirer, comment distinguer la diversité sensible, telle qu'elle se différencie affectivement, et la diversité des formes symboliques dont on ne peut savoir d'où elle provient, tant que n'apparaît pas l'enracinement affectif de la symbolisation ? Cest en résolvant ce probleme de la double transposition du dynamisme et de la diversité spécifiquement sensible dans l'aperception rationnelle qu'il est possible de formuler l'articulation de la double aperception, affective et rationnelle, par l'horizon symbolique de l'action. Par exemple, le mythe apparaît là oủ l'action, demeurant rare et marginale, ne peut offrir qu'une altérité actuellement illimitée dans l'espace-temps (et non pas virtuellement, comme dans le symbole), de telle sorte que cette action se dissout rapidement en une affectivité toute-puissante et anonyme au lieu d'affirmer la genèse intersubjective de la subjectivité qui, en s'incarnant, permettrait de faire surgir le corps susceptible de sous-tendre l'expression rationnelle de la pensée là même où cette pensée ne s'affirme que comme pure « action de l'esprit ». En partant du mythe, où le langage se trouve écartelé entre une action évanescente et une pure pensée qui s'assimile celle-ci, il est possible d'enchaîner avec la mythologie où s'amorce l'expression rationnelle de la pensée correspondant à l'expression cosmobiologique de la nature ou, encore, d'aborder la science moderne comme un processus de systématisation de cette ex pression rationnelle de la pensée par la mise à jour du fondement corporel de la nature, etc. Les diverses formes symboliques surgissent, alors, de la symbolisation définissant l'homme par l'articulation de son aperception duale qui s'oriente diversement, par l'affectivité et la raison, en polarisant le sensible et l'intelligible selon des expressions propres à chacune de ces orientations (l'affectivité symbolisante, l'animal raisonnable). 
Kant (Critique de la raison pratique) l'inverse au profit de la raison dans le sentiment moral, il est possible de remettre en question la distorsion rationaliste que Kant fait subir à la différenciation sensible et, en particulier, à l'affectivité mais en préservant la complexité du rapport entre le sensible et l'intelligible par laquelle s'énonce la liberté.

En conclusion, une double aperception affective et rationnelle de l'homme permet de rendre compte du rapport entre le sensible et l'intelligible de façon réellement immanente, dans la mesure où l'on tient compte de ses transformations à travers différentes conceptions de l'homme, tel qu'il se deffinit comme culture. Ainsi, l'homme, comme animal raisonnable du monde grec, identifie l'intelligible à la raison et décentre la sensibilité de son dynamisme essentiellement affectif en identifiant cette sensibilité aux sens et à une irrationnalité. Par contre, l'affectivité symbolisante apparaît, à notre époque, en identifiant la sensibilité à son dynamisme essentiellement affectif tout en tenant compte du décentrement a priori de la raison qu'est le symbole. Aussi, l'affectivité paradoxalement rationaliste de Henry et l'animal symbolique de Cassirer apparaissent comme des mélanges conjuguant des réalités culturelles hétéroclites. Qu'une culture mette en oeuvre de façon systématique la raison, ne signifie pas que la culture, ni même cette culture, s'y réduisent, qu'il s'agisse de celle des Grecs ou de la nôtre, ou qu'il s'agisse encore d'un même élan de civilisation départageant ses moments culturels. Le rapport à l'être et le rapport à son être ne sont pas le rapport à la culture, ni même le rapport à sa culture. Car une question demeure : qui énonce l'être et/ou son être? L'homme ou la métaphysique que produit l'homme ${ }^{37} \ldots$ en Occident?

\section{Département de philosophie \\ Université d'Ottawa}

37. Ce dernier cas s'affirme d'emblée par une inversion où la métaphysique se trouve au fondement de l'homme et où, en ayant exclu l'homme lui-même de façon toute aussi a priori, le discours sur l'homme se deroule sans l'homme afin que tout homme concret, au sens empirique, y trouve un contexte purement spéculatif. L'action prend alors un sens purement nominal puisqu'il n'est question, de fait, que d'activité où l'analyse structurale rend compte de l'acte indépendamment de celui qui le produit, même si, à la limite, celui qui analyse spéculativement et celui qui agit corporellement peuvent « coïncider » en procédant d'une même individualité, fondant leur exclusion mutuelle et leur indépendance respective. 